Mayo 2020

\title{
Publicación de artículos académicos: buenas prácticas y recomendaciones para la redacción y envío a revistas científicas
}

\author{
Rafael Pedraza- Jimenez \\ Universitat Pompeu Fabra \\ rafael.pedraza@upf.edu \\ ORCID: 0000-0002-6918-6910
}

DOI: $10.31009 /$ methodos.2020.i01.04

Pedraza - Jimenez, R. (2020). Publicación de artículos académicos: buenas prácticas y recomendaciones para la redacción y envío a revistas científicas. En: Lopezosa, C.;

Díaz-Noci, J.; Codina, L. (ed.). Anuario de Métodos de Investigación en Comunicación Social, n.1 (p.38-49). Barcelona: DigiDoc-Universitat Pompeu Fabra

Esta obra está bajo una Licencia

Creative Commons Atribución

4.0 Internacional.

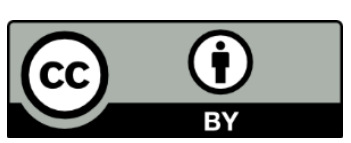






\title{
Publicación de artículos académicos: buenas prácticas y recomendaciones para la redacción y envío a revistas científicas
}

\author{
RAFAel PedRaZA- Jimenez \\ Universitat Pompeu Fabra \\ rafael.pedraza@upf.edu \\ ORCID: 0000-0002-6918-6910
}

\section{RESUMEN}

En el contexto académico actual la publicación de artículos de investigación en revistas científicas se ha convertido en un requerimiento, no solo para desarrollar una carrera académica, sino incluso para la superación de diferentes estudios de postgrado. A pesar de ello, continúan siendo escasos los recursos que guían y orientan a las personas que deben abordar la ardua tarea de publicar. Este artículo pretende contribuir a llenar este vacío. A tal fin, recopila recomendaciones y buenas prácticas que intentan ayudar, principalmente -aunque no exclusivamente-, a los investigadores noveles a maximizar sus posibilidades de publicar con éxito en una revista científica. De este modo, en este trabajo se abordan aspectos relacionados con: la redacción de los artículos científicos; la selección de revistas apropiadas para su publicación; el proceso de envío del artículo a revistas científicas; el proceso de evaluación del artículo por parte de una revista; y el procedimiento de respuesta a los comentarios de los revisores de la revista.

\section{PALABRAS CLAVE}

Redacción científica, Redacción académica, Artículos científicos, Artículos académicos, Publicación de artículos académicos, Publicación de artículos científicos, Estructura de un artículo académico.

Publication of academic articles: good practices and recommendations for writing and sending to scientific journals
Publicació d'articles acadèmics: bones pràctiques i recomanacions per a la redacció d'articles d'investigació i l'enviament a revistes científiques

\begin{abstract}
In the current academic context, the publication of research articles in scientific journals has become a requirement, not only to develop an academic career, but also to overcome different postgraduate studies. Despite this, the resources that guide people who must tackle the arduous task of publishing remain scarce. This article aims to contribute to filling this gap. To this end, it presents recommendations and good practices that are useful, mainly -although not exclusively-, to new researchers to maximize their chances of successfully publishing in a scientific journal. In this way, this work addresses aspects related to: the writing of scientific articles; the selection of appropriate journals for publication; the process of sending the article to scientific journals; the article evaluation process by a scientific journal; and the procedure for responding to the comments of the journal reviewers.
\end{abstract}

\section{KEYWORDS}

Scientific writing, Academic writing, Scientific articles, Academic articles, Publication of academic articles, Publication of scientific articles, Structure of an academic article.

\begin{abstract}
RESUM
En el context acadèmic actual la publicació d'articles d'investigació en revistes científiques s'ha convertit en un requeriment, no només per desenvolupar una carrera acadèmica, sinó fins i tot per a la superació de diferents estudis de postgrau. Tot i això, continuen sent escassos els recursos que guien i orienten a les persones que han d'abordar l'àrdua tasca de publicar. Aquest article pretén contribuir a omplir aquest buit. Amb aquesta finalitat, recopila recomanacions i bones pràctiques que intenten ajudar, principalment -encara que no exclusivament-, als investigadors novells a maximitzar les seves possibilitats de publicar amb èxit en una revista científica. D'aquesta manera, en aquest treball s'aborden aspectes relacionats amb: la redacció dels articles científics; la selecció de revistes apropiades per a la seva publicació; el procés d'enviament de I'article a revistes científiques; el procés d'avaluació de l'article per part d'una revista científica; i el procediment de resposta als comentaris dels revisors de la revista.
\end{abstract}

\section{PARAULES CLAU}

Redacció científica, Redacció acadèmica, Articles científics, Articles acadèmics, Publicació d'articles acadèmics, Publicació d'articles científics, Estructura d'un article acadèmic. 


\section{Introducción}

La publicación de un artículo en una revista científica es una tarea compleja que requiere el conocimiento de ciertos procesos de trabajo. La redacción del texto, la idoneidad de la revista escogida para su publicación, la presentación del trabajo al editor de la revista, o la interacción con los revisores, son solo algunos de los aspectos que pueden determinar que un artículo sea o no publicado.

En este texto se abordan todos estos aspectos. Se ha estructurado de manera que cada uno de sus apartados se corresponda con las diferentes fases que es necesario abordar para la publicación de un trabajo. Es decir, desde la concepción del artículo hasta su publicación final en una revista académica.

Se trata, por tanto, de una guía que pretende ayudar, principalmente -aunque no exclusivamente-, a los investigadores noveles cuando abordan la ardua tarea de publicar sus primeros artículos académicos.

\section{Consideraciones previas a la redacción del artículo}

Antes de abordar la redacción de un artículo, convendría que el investigador, sobre todo si es un investigador novel, reflexionara sobre las siguientes cuestiones:

a) ¿Qué es más conveniente publicar individualmente o en equipo con otros investigadores?

Un investigador puede optar indistintamente por publicar individualmente o con otros autores. No obstante, en las etapas iniciales de la carrera académica publicar con otros investigadores puede ser muy instructivo y útil.

Instructivo porque la colaboración con investigadores más experimentados permitirá conocer y aprender sus métodos de trabajo. Además, estos investigadores ayudarán a poner en valor las aportaciones más novedosas y valiosas del artículo. Esta tarea, que resulta compleja cuando se carece de experiencia investigadora, es fundamental para superar con éxito el proceso de revisión de una revista académica y publicar nuestros trabajos.

Aunque trabajar colaborativamente presenta otras muchas ventajas. De entrada, la carga de trabajo será más liviana, tanto durante el desarrollo de la investigación como en su posterior difusión (en forma de artículos, comunicaciones a congresos, etc.). Además, los investigadores noveles que colaboren con investigadores más experimentados también se beneficiarán de la visibilidad de estos últimos. Es decir, la reputación de los investigadores más consolidados probablemente ayudará a que el trabajo publicado tenga más posibilidades de ser leído por sus seguidores y colegas.

Por tanto, el trabajo y la publicación en equipo ofrecen a los nuevos investigadores ventajas y oportunidades que convendría tener muy presentes. Ahora bien, si se opta por realizar un trabajo en coautoría...

\section{b) ¿Cuántos investigadores pueden firmar un} artículo?

No existe un número máximo de coautores. Pueden firmar tantos autores como hayan sido necesarios para realizar la publicación. De hecho, el número de autores varía de unas disciplinas a otras. Por ejemplo, en el ámbito de las humanidades el número de coautores es más reducido que en los ámbitos de las ciencias experimentales y de la salud.

Obsérvese que, en ocasiones, en algunas comunidades científicas se establece un número recomendado de firmantes. Este tipo de prácticas suelen estar motivadas por razones administrativas. Por ejemplo, antaño en España se recomendaba que el número máximo de firmantes de un artículo fuera de tres. Esta recomendación no se debía a ninguna razón objetiva. Se había establecido así porque las agencias nacionales para la evaluación y la acreditación de investigadores valoraban peor los trabajos con más de tres autores. Actualmente, este tipo de prácticas van desapareciendo.

Llegados a este punto, si se decide trabajar en equipo...

\section{c) ¿A quién se considera autor del artículo?}

Las pautas COPE (Committee on Publication Ethics) son muy claras a este respecto. Establecen que son autores quienes participan en el desarrollo de la investigación y en la redacción del artículo (Codina, 2018).

Se considera que una persona participa en la investigación cuando contribuye a:

- La concepción y diseño de la investigación, o...

- La adquisición de datos, $\mathbf{o . . .}$

- El análisis e interpretación.

Nótese que una persona solo necesita cumplir uno de los tres requerimientos anteriores (y no todos ellos) para participar en la investigación. Además, deberá contribuir a la redacción del artículo, satisfaciendo al menos una de las condiciones siguientes:

- Participar en la redacción del artículo, o...

- Participar en la revisión del artículo.

De este modo, resulta sencillo determinar quiénes 
deben ser considerados autores de un artículo. No obstante, el desarrollo de trabajos en coautoría genera una nueva problemática: cuando hay varios autores...

\section{d) ¿Cómo se establece el orden de firma?}

No existe un criterio único para establecer el orden de firma. Con frecuencia, el orden de firma suele reflejar la dedicación y el esfuerzo que los autores han invertido para que el artículo salga adelante. De esta manera, el primer autor suele ser la persona que lleva la iniciativa y redacta la primera versión del trabajo.

No obstante, se ha de tener presente que existen diferentes tradiciones en relación al orden de firma. Por ejemplo, también es muy habitual que el primer autor sea la persona que llevó la iniciativa en la redacción del trabajo, y que el último autor sea la persona que concibe, coordina y/o revisa la versión final del artículo (ocupando así la primera y la última posición de firma autores con roles muy importantes).

En algunos dominios de conocimiento incluso se adopta un orden de firma alfabético. Por tanto, es recomendable identificar la tradición seguida en nuestro ámbito y adaptarnos a ella. Eso sí, siguiendo criterios éticos que garanticen el reconocimiento de la labor realizada por todas las personas que han contribuido al desarrollo del artículo.

\section{Redacción de un artículo académico}

Una vez se ha decidido trabajar en equipo se procederá a la planificación y redacción del trabajo.

\subsection{Planificación del artículo}

Será muy importante decidir quién será el autor que redacte la primera versión del artículo. El hecho de que un solo autor asuma la redacción de un primer borrador del artículo tiene diversas ventajas:

- Agiliza la redacción: disponer de la primera versión del documento dependerá exclusivamente de la agenda de uno de sus autores

- Propicia que la redacción y la presentación de ideas sea coherente y uniforme: se evita así la creación de "documentos tipo Frankenstein", es decir, realizado con fragmentos de distintos autores $\mathrm{y}$, en consecuencia, con distintos estilos tanto en su redacción como en su estructura.

También habrá que determinar qué rol tendrán el resto de los autores:

- Extracción y/o procesamiento de datos

- Revisión del texto en profundidad
- etc.

Por último, habrá que establecer un calendario realista de trabajo.

\subsection{Estructura del artículo}

Cuando redactamos nuestros primeros artículos suele ser útil elaborar un esquema inicial. Una de las estructuras más habituales (aunque no la única) en artículos de investigación es:

- Título, resumen y palabras clave

- Introducción

- Objetivos

- Metodología

- Resultados

- Discusión y Conclusiones

- Referencias

No obstante, se recomienda que el orden de redacción de estos apartados no sea secuencial. Normalmente la elaboración del artículo resulta más sencilla cuando se comienza con la redacción de los objetivos, es decir, planteando en primer lugar lo que pretende nuestra investigación. El apartado de objetivos suele detallar tanto el objetivo general de la investigación como los diferentes objetivos específicos en los que se concreta el objetivo general para su consecución. Recuérdese además que estos objetivos se suelen redactar utilizando el infinitivo, por ejemplo: este trabajo pretende conocer / estudiar / identificar / hallar / explorar / etc. Asimismo, el apartado de objetivos introduce con frecuencia información sobre el alcance y las limitaciones del trabajo. Un subapartado de alcance y limitaciones permite al investigador dejar constancia, por ejemplo, de aspectos que se han preferido no abordar en el estudio.

Después de los objetivos es aconsejable abordar la redacción de la metodología. En ella se explica cómo se pretenden alcanzar los objetivos propuestos, es decir, con qué materiales, programario y métodos de investigación, entre otros. Además, debe incluir aspectos tales como el procedimiento para la selección del objeto de estudio o las fechas de análisis. Idealmente el apartado de metodología debe permitir a terceros investigadores conocer las condiciones exactas en las que se desarrolló nuestra investigación $\mathbf{y}$, en algunos casos, incluso permitirles reproducir y contrastar nuestros resultados si así lo desearan. Al igual que en el caso anterior, en este apartado también se puede incluir un subapartado con limitaciones del trabajo. En él se podrán detallar los problemas metodológicos que hayan surgido durante la investigación. 
En tercer lugar, es conveniente proceder a la redacción del apartado de resultados. En ellos se detallarán los resultados obtenidos tras la aplicación de los distintos métodos y técnicas explicados en el apartado de metodología. En el apartado de resultados se debe evitar incluir una explicación de estos. Este es un error que frecuentemente cometen los investigadores noveles. Esta explicación debería hacerse en el apartado de discusión.

Tras los resultados, es apropiado redactar los apartados de discusión y conclusiones. En función de la revista, e incluso de las preferencias de los investigadores, encontraremos ambos apartados por separado o unidos. En cualquier caso, se ha de tener presente que el apartado de discusión incluirá la interpretación de nuestros resultados y la comparación de estos con resultados de investigaciones previas.

En cuanto a las conclusiones, en ellas se constatará el grado de cumplimiento de los objetivos propuestos en el trabajo. También se presentará una reflexión general sobre toda la investigación. A menudo esta reflexión se centra en exceso en los resultados. Conviene que esta reflexión contemple aspectos de todo el trabajo, como, por ejemplo, la utilidad que han tenido las diferentes metodologías aplicadas, etc.

La redacción de la introducción es aconsejable abordarla una vez se han redactado los apartados principales del artículo, en ella se describirá:

- El contexto en el que se desarrolla la investigación presentada.

- Los antecedentes y el marco teórico, es decir, los autores y trabajos previos que han abordado la problemática que estamos estudiando, o bien han fundamentado la disciplina en la que se desarrolla nuestra investigación.

- La introducción podría terminar con un último párrafo que explique al lector cómo está estructurado todo el trabajo.

Por último, se procederá a la redacción del título, las palabras clave y el resumen. En relación con el título, este debe ser claro, descriptivo y conciso. Téngase en cuenta que, normalmente, el título es el elemento utilizado por los investigadores para seleccionar un trabajo entre una larga lista de resultados ofrecidos por las bases de datos y los buscadores académicos. En cuanto a las palabras clave, se recomienda introducir al menos cinco. Además, conviene que representen conceptos tanto generales (como el nombre de disciplinas) como específicos (aspectos concretos en los que se centra la investigación realizada). Por último, el resumen describirá brevemente todo el trabajo realizado. Una técnica sencilla para conseguir

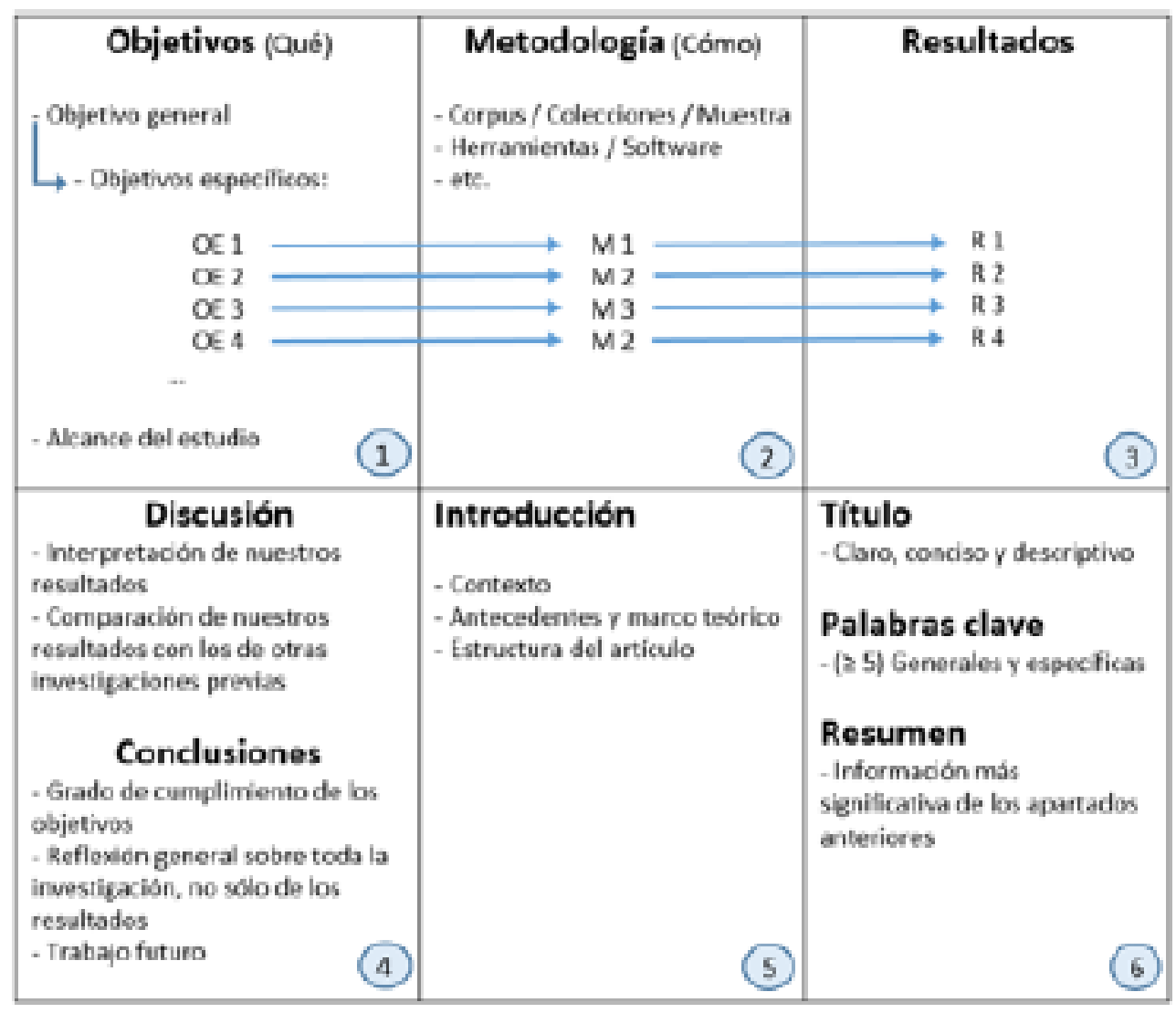

Figura 1. Propuesta de fases para la redacción de un artículo académico. Fuente: elaboración propia. 
un buen resumen es:

- Incluir una frase que presente el objetivo general del trabajo.

- Añadir una o dos frases que concreten cuál es el objeto de estudio abordado y qué metodologías se han utilizado para su análisis.

- Explicar con una frase cuál es el resultado más importante que se ha obtenido.

- Terminar el resumen con una o dos frases que expliquen la relevancia del resultado y si se ha alcanzado el objetivo propuesto en la investigación.

La figura 1 sintetiza la estructura y el proceso de redacción de un artículo de investigación estándar.

Téngase en cuenta que esta estructura, a pesar de ser muy utilizada, puede variar -normalmente levementeen función del tipo de investigación desarrollada, y también en función de las preferencias de formato de la revista en la que se decida publicar.

\subsection{Redacción del artículo}

Una vez detallada la estructura básica de un artículo de investigación, se enumeran algunas recomendaciones para la redacción del artículo:

1. Escribir frases cortas: idealmente que no superen las dos líneas de longitud. Escribir utilizando frases cortas no es natural. Por ello, aconsejamos a los investigadores noveles que redacten la primera versión de su artículo con naturalidad, sin controlar la longitud de las oraciones que utilizan. Una vez terminado, el investigador podrá revisar y reducir con facilidad la longitud de las frases que ha escrito. Con frecuencia, en este proceso de revisión basta con identificar las frases muy largas y sustituir las comas que en ellas aparecen por puntos. La aplicación de esta práctica irá entrenando al investigador en este tipo de escritura, y le ayudará, progresivamente, a interiorizarla.

2. Usar conectores entre párrafos: otro problema que habitualmente se detecta en los primeros trabajos de un investigador es la falta de coherencia y relación entre los distintos párrafos del texto. Una solución a esta problemática es el uso de conectores al comienzo -o al final- de cada párrafo. De hecho, la creación y el mantenimiento de un listado de conectores para su consulta durante el proceso de redacción ayudará a solventar este problema. Algunos ejemplos de conectores utilizados frecuentemente en artículos académicos son: por tanto, de este modo, asimismo, por otro lado, además, no obstante, etc.

3. Relacionar los diferentes apartados del artículo entre sí: la falta de conexión entre los diferentes apartados de un artículo (por ejemplo, entre la introducción y los objetivos, o entre los objetivos y la metodología, etc.) puede dificultar su comprensión. Por ello, finalizar -o comenzar- los diferentes apartados haciendo una breve referencia al contenido que sigue -o precede, según sea el caso- facilitará la realización de una lectura continua de nuestros textos. En este sentido, pueden ser útiles expresiones tales como:

«Especificados los objetivos de este trabajo, procedemos a detallar los métodos utilizados para su consecución.〉

O también:

«A continuación, se presentan los resultados alcanzados tras la aplicación de los métodos descritos.〉>

4. Redactar en estilo impersonal: en los artículos académicos se utilizarán, normalmente, formas verbales impersonales. Debería evitarse el uso de la primera persona del singular. Aunque sí podemos utilizar la primera persona del plural, incluso cuando el artículo está firmado por un único autor. En todo caso, se recomienda no abusar de esta forma verbal y utilizar oraciones impersonales cuando sea posible.

5. Evitar la repetición de contenido: los nuevos investigadores repiten con frecuencia sus ideas en los distintos apartados de sus artículos. Esta praxis debería evitarse. La presentación del contenido y las ideas en un texto académico debería ser secuencial y, en consecuencia, sin repeticiones. Tan solo los apartados de discusión y conclusiones deberían recuperar ideas ya expuestas en el texto, con la finalidad de completarlas mediante su interpretación o reflexión.

6. Prescindir de opiniones y afirmaciones categóricas no fundamentadas: un texto que introduce opiniones o afirmaciones no contrastables difícilmente superará la evaluación de sus revisores. Por tanto, se recomienda evitar su uso en artículos académicos o, en todo caso, acompañarlas con datos o referencias que avalen su veracidad.

7. Constatar las limitaciones de la investigación realizada: si la investigación realizada presenta limitaciones es conveniente declararlo. Ocultarlo con la esperanza de que pasen inadvertidas para los revisores no es una buena praxis. Por un lado, porque un evaluador experto probablemente las detectará, y podría intuir que se han ocultado a propósito (lo que no favorecería su opinión sobre nuestro trabajo). Por otro, porque la omisión de las limitaciones dificulta la interpretación y reproducción del trabajo y, además, podría conducir a conclusiones erróneas a nuestros lectores.

8. Introducir tablas, figuras y listas cuando el texto lo requiera: un artículo debe presentar la información de la manera más clara y concisa posible. El uso de tablas, 
figuras y listas contribuyen a este fin. Si la comprensión de ciertos párrafos de nuestro texto resulta compleja, convendrá reflexionar sobre la idoneidad de presentar su contenido de una manera alternativa. Por ejemplo, en ocasiones, podría sustituirse la enumeración de diversas cantidades numéricas (porcentajes, etc.) en un párrafo por una tabla. Asimismo, en muchas ocasiones, resultará más claro para un lector la presentación de diversos elementos en forma de lista, en lugar de utilizar los dos puntos (:) y a continuación diversas oraciones separadas por punto y coma (;).

9. Utilizar pies o encabezamientos descriptivos y bien enumerados en tablas y figuras: todas las tablas y figuras del texto deben ir acompañadas de un texto descriptivo que ayude al lector a entender su contenido.

10. Cuidado con las relaciones "causa-efecto" difícilmente demostrables: en ocasiones los resultados obtenidos en nuestra investigación nos pueden inducir a formular relaciones de causa-efecto. Si así lo hiciéramos, nuestro texto debería demostrar esa relación con datos o informaciones obtenidas en nuestra investigación.

Ahora bien, ¿qué ocurre si nuestros resultados apuntan a una probable relación de causa-efecto que es difícilmente demostrable? En estos casos se puede optar por una realizar una exposición secuencial de hechos.

Por ejemplo, en su tesis doctoral, la investigadora Laura Pérez-Altable (2016) explica que antes de diciembre de 2010 se habían producido diversas inmolaciones en señal de protesta en Túnez. No obstante, fue la inmolación de Mohammed Bouazizi, en diciembre de 2010, la que inició la primera ola de protestas sociales que acabaría en lo que hoy conocemos como la Primavera Árabe.

Esta investigadora explica en su tesis doctoral que Bouazizi, a diferencia de otras personas que se habían inmolado con anterioridad, conocía a activistas sociales muy populares en los medios sociales. También relata que estos activistas dieron a conocer en sus plataformas sociales el caso de Bouazizi y llamaron a la acción a la ciudadanía.

Ahora bien, en ningún momento la Dra. Pérez-Altable establece en su investigación una relación de causa-efecto entre "la relación personal existente entre Bouazizi y los activistas sociales" y "la llamada a la acción social y su masivo seguimiento". En su lugar, expone de manera secuencial los hechos descubiertos y acontecidos.

11. Revisar y corregir las erratas: las erratas y los errores ortográficos denotan dejadez y apresuramiento por parte de los autores. Un texto con este tipo de errores difícilmente será aceptado por el editor o los revisores de una revista.

12. Presentar las referencias de manera homogénea: las revistas académicas facilitan a los autores instrucciones sobre el formato que deben tener los artículos a ellas enviados. En estas instrucciones se detalla el formato específico de las referencias incluidas en el artículo. Normalmente, resulta fácil homogeneizar y adaptar las referencias de un trabajo a las exigencias de una revista, especialmente si los autores han utilizado gestores de referencias, como por ejemplo Mendeley o RefWorks.

\section{Selección de una revista académica para publicar}

La elección de la revista en la que se publicará nuestra investigación no es una tarea sencilla. A continuación, se enumeran algunos de los aspectos que habría que valorar antes de enviar nuestro trabajo:

1. Temática: es fundamental que el investigador consulte la temática de interés para la revista. Esta información suele estar disponible en su sitio web. Normalmente, se especifica en secciones que pueden presentar títulos tales como: "Enfoque"; "Temática"; "Ámbito"; etc. En ocasiones, esta información aparece directamente en el apartado de "Información para los autores".

Para un investigador novel, que aún no conoce bien las revistas de su ámbito de trabajo, es esencial consultar la temática en la que se centra una revista antes de enviar su artículo. De lo contrario, el autor podría recibir, semanas o meses después de realizar el envío, una respuesta del editor rechazando el artículo e indicando que el trabajo no encaja con las líneas de interés de la revista.

Evitar este tipo de retrasos en la publicación de un trabajo siempre es positivo, más aún si los autores son investigadores que están realizando una tesis por compendio, pues, con frecuencia, están sometidos a fuertes restricciones temporales para cumplir con los plazos establecidos en sus programas de doctorado.

Por último, además de consultar la sección o apartado donde se especifica la temática que aborda la revista, es muy conveniente ojear los últimos números publicados por la revista en la que deseamos publicar. De este modo conoceremos los temas, e incluso algunos de los enfoques metodológicos, que actualmente son de interés para la publicación.

2. Idioma: la publicación de textos en un idioma distinto a nuestra lengua materna puede ser problemática. Si no somos angloparlantes, y la revista en 
la que deseamos publicar solo acepta trabajos en inglés, convendrá que nuestro texto sea revisado por un traductor nativo. Con frecuencia, tener un excelente nivel de inglés no es suficiente para que un texto pase el filtro de un editor o revisor nativos. En consecuencia, la publicación en una lengua diferente a nuestra lengua materna suele suponer que el investigador ha de disponer de recurso económicos para pagar una traducción.

3. Índices y bases de datos: uno de los indicadores más utilizados para valorar la calidad de una revista es su presencia en diferentes bases de datos o índices. En la actualidad, Web of Sciences (WoS) y Scopus están consideradas las dos principales bases de datos científicas a nivel internacional. Es decir, la comunidad científica considera que, en términos generales, las revistas científicas de mayor calidad están indexadas en estas dos bases de datos.

Por tanto, un criterio que podemos utilizar a la hora de escoger una revista para publicar es si esta tiene presencia en alguna de estas bases de datos. Téngase en cuenta además que, tanto WoS como Scopus utilizan índices que informan a su vez de la calidad de cada revista dentro de la propia base de datos. Es decir, ambas bases de datos establecen un ranking de las revistas que indexan. La calidad de la revista según estas bases de datos también puede ser tenida en cuenta a la hora de escoger la revista en la que publicar.

Ahora bien, un investigador novel debería ser realista, y ser consciente de que cuanta más calidad y/o impacto tiene una revista más difícil es publicar en ella. Por tanto, normalmente será muy difícil que nuestras primeras investigaciones sean aceptadas para su publicación en revistas de gran prestigio a nivel internacional.

Por otro lado, también es importante señalar que la publicación en revistas que no están indexadas en las principales bases de datos no está en absoluto desaconsejada. Por un lado, porque en algunas disciplinas existen revistas con un enfoque más humanístico y/o divulgativo que no son de interés para las bases de datos mencionadas. Por otro, porque muchas revistas no indexadas en estas bases de datos gozan de una gran popularidad y prestigio en ámbitos geográficos o temáticos reducidos. Tanto es así, que es fácil encontrar revistas no indexadas en WoS o Scopus pero cuya calidad está reconocida en los índices de calidad elaborados por organismos y/o comunidades científicas concretas, a nivel de país, región, disciplina, etc.

4. Extensión de los artículos: la extensión máxima de los artículos que acepta una revista es otro de los criterios que hemos de tener en cuenta antes de enviar nuestro artículo. Ciertamente, el hecho de que cada vez haya más publicaciones en línea está ayudando a acabar con estas restricciones. No obstante, aun son muchas las revistas con versión impresa, e incluso estrictamente digitales, que establecen un número máximo de palabras o páginas. Por tanto, hemos de estar seguros de que nuestro texto cumple con este requerimiento antes de su envío. Es más, si el texto que se desea enviar es extenso, y la aceptación del artículo requiere una severa reducción del mismo, quizás convendría contemplar la posibilidad de enviar el artículo a otra revista.

5. Tipo de publicaciones: en el sitio web de las revistas se encontrará información sobre el tipo de artículos que publican, como, por ejemplo, artículos de investigación, revisión, etc. Es aconsejable no enviar a una revista artículos que no coincidan con el tipo de publicación que aceptan, ya que el trabajo tendrá muchas posibilidades de ser rechazado.

6. Restricciones del copyright: otro aspecto sobre el que informan las revistas es el tipo de licencia bajo el cual se distribuyen sus contenidos. Actualmente, existen revistas que distribuyen sus contenidos en acceso abierto (Open Access, OA), revistas con copyright pero que permiten la publicación de algunos artículos en acceso abierto (previo pago), y revistas que solo publican artículos con copyright. Como autores, hemos de ser conscientes de estas diferencias y de decidir a qué tipo de revista enviaremos nuestros trabajos. Además, si la investigación que se presenta ha sido financiada por una institución pública, es posible que exista la condición de difundirla a través de revistas de acceso abierto.

7. Periodicidad de la revista y tiempo promedio del proceso de revisión: la periodicidad de una revista varía enormemente de unos títulos a otros. Lo mismo ocurre con los tiempos de revisión de cada título. Afortunadamente, a través de sus sitios web las revistas suelen poner a disposición de sus potenciales autores ambas informaciones. Aunque a priori ambos tiempos puedan no parecer un factor muy importante para escoger la revista en la que publicar, en ocasiones, sí es un criterio relevante (e incluso crítico). Piénsese, por ejemplo, en un estudiante de doctorado que esté realizando su tesis por compendio de publicaciones, o en el caso de un investigador que deba presentar los resultados de una investigación o proyecto en un plazo de tiempo muy ajustado.

8. Números especiales: por último, las revistas también informan en su web de los números especiales que tienen programados. Los números especiales son volúmenes que se dedican a una temática específica. Si nuestra investigación se corresponde con la temática de un número especial es buena idea presentarla para su publicación en el mismo. Téngase en cuenta que la especialización de un número supone una reducción del número de autores que están en condiciones de 


\begin{tabular}{|c|c|c|c|c|c|c|c|c|c|c|}
\hline Revista & $\begin{array}{l}\text { Indice / If } \\
\text { Wos / Sco }\end{array}$ & $\begin{array}{c}\text { Cuartil } \\
\text { Wos / Sco }\end{array}$ & Temáticas & Idioma & $\begin{array}{l}\text { Extensión } \\
\text { articulos }\end{array}$ & $\begin{array}{c}\text { Tipos } \\
\text { articulos }\end{array}$ & $\begin{array}{c}\text { Cosvright } \\
\text { / oa }\end{array}$ & $\begin{array}{c}\text { Coste } \\
\text { oA }\end{array}$ & Periodiclidad & $\begin{array}{l}\text { Tiempo de } \\
\text { revisibin }\end{array}$ \\
\hline & & & & & & & & & & \\
\hline & & & & & & & & & & \\
\hline & & & & & & & & & & \\
\hline & & & & & & & & & & \\
\hline & & & & & & & & & & \\
\hline
\end{tabular}

Figura 2. Características de las revistas candidatas a recibir un artículo. Fuente: elaboración propia.

presentar sus trabajos y, en consecuencia, un aumento de las posibilidades de publicación de los trabajos presentados.

Registrar (en una tabla, base de datos, etc.), para su consulta y comparación, las características indicadas anteriormente sobre las revistas puede ayudarnos a decidir la publicación más apropiada a la que enviar cada investigación. La figura 2 muestra una tabla que, idealmente, recopilaría y evidenciaría las características de las revistas candidatas a las que se podrían enviar los trabajos realizados.

\section{Envío de un artículo a una revista académica}

Una vez se haya decidido la revista a la que se enviará el artículo se procederá a las siguientes comprobaciones:

- Se revisará el texto para estar seguros de que está adaptado correctamente al formato solicitado por la revista.
- Se revisarán las referencias para constatar que se han seguido las normas de citación exigidas por la revista.

Además, será necesario decidir cuál de los autores actuará como contacto durante el proceso de revisión, es decir, se elegirá al autor que asumirá el rol de corresponding author. Este autor normalmente asume la responsabilidad de enviar una carta de presentación del artículo denominada cover letter. Asimismo, suele ser el responsable de aceptar, en nombre de todos los autores, las condiciones de copyright de la revista. También es el autor que interactúa con los revisores durante el proceso de revisión.

A continuación, a modo de ejemplo, la figura 3 muestra una cover letter. Como puede apreciarse, este tipo de documentos deben contener tres informaciones: la presentación y puesta en valor de la investigación realizada; la declaración de la originalidad del trabajo y de la predisposición de los autores a aceptar las condiciones de copyright de la revista; y, por último, la información de contacto del corresponding author.

Antes de proceder al envío, los investigadores nóveles

Dear Professor XXX:
\begin{tabular}{|l|}
\hline I am pleased to submit our original manuscript 'XXX' by XX et al. for consideration for publication as \\
a research article in Online Information Review. \\
We believe this manuscript is suitable for publication as the focus of the research reflects the aims and \\
scope of your journal and the reviewer of a previously submitted paper expressed interest in the line of \\
research it explores. \\
Our manuscript presents a semi-automatic model for updating controlled vocabularies through the use \\
of a text corpus and the mining of query logs. Today, controlled vocabularies play an important role in \\
information retrieval. Numerous studies have shown that conceptual searches based on vocabularies \\
are more effective than keyword searches, at least in certain contexts. Consequently, new ways must \\
be found to improve controlled vocabularies.
\end{tabular}

\begin{tabular}{l} 
All the authors have approved the manuscript and agree to its submission to Online Information \\
Review. Moreover, the manuscript is the authors' original work and has not been published or \\
simultaneously submitted elsewhere. \\
Please address all correspondence to: \\
XXX \\
Department of Communication. Universitat Pompeu Fabra, \\
Campus de la Comunicació, Roc Boronat 138, 08018 Barcelona, Spain \\
Phone: +34 \\
E-mail: xxxx@upfedu \\
\hline
\end{tabular}

Figura 3. Ejemplo de cover letter. Fuente: elaboración propia. 
deberían reflexionar sobre el nombre que utilizarán para firmar su artículo. Es muy importante decidir cuál será nuestra "autoría normalizada", y utilizarla siempre para firmar nuestros trabajos. Los autores que no normalizan su nombre corren el riesgo de no ser identificados adecuadamente por las bases de datos, lo que puede suponer a su vez problemas de citación para estos autores. La iniciativa iralis (International Registry for Authors: Links to Identify Scientists) ayuda a los autores y les asesora en el proceso de normalización de sus nombres.

Asimismo, durante el proceso de envío del artículo es muy probable que la revista solicite el código ORCID de los autores. El código ORCID (Open Researcher and Contribution ID) ayuda a identificar de manera unívoca a un investigador y su producción científica. Con frecuencia, los investigadores que no disponen de él tienen que registrarse y obtener uno para poder formalizar el envío de sus trabajos.

Por último, destacamos una cuestión muy importante en relación con el envío de artículos que los nuevos investigadores han de tener muy presente. Los autores no deben enviar su trabajo a más de una revista a la vez. Hacerlo se considera una mala praxis, además de poco ético. Por tanto, los autores escogerán la revista que consideren más pertinente para la publicación de su trabajo, lo enviarán a ella, y esperarán su respuesta antes de proceder a un nuevo envío del mismo artículo a otra revista.

\section{Proceso de evaluación de un artículo académico}

Una vez enviado el artículo comenzará su proceso de evaluación. Este proceso consta de dos fases:

- Evaluación editorial

- Revisión por pares

En la evaluación editorial el editor de la revista realizará una primera valoración del trabajo. En esta fase normalmente se comprueba si la temática del artículo encaja en las líneas de interés de la revista, si la redacción del trabajo es apropiada, y si el trabajo es original. Si la evaluación editorial es favorable el editor procede a enviar el trabajo a los revisores (dos o tres habitualmente) para que analicen con más profundidad el trabajo presentado. Si el trabajo enviado no pasa la evaluación editorial el editor escribirá al "corresponding author" comunicándole el rechazo de la publicación y los motivos del mismo.

En cuanto a la revisión por pares, esta puede ser abierta, a simple ciego o a doble ciego. En la revisión abierta los autores y los revisores conocen sus respectivas identidades. En la revisión a simple ciego los revisores conocen la identidad de los autores, pero estos desconocen a los revisores. Por último, en la revisión a doble ciego todos, autores y revisores, son anónimos. En este momento, lo más habitual son las revisiones por pares a simple y a doble ciego. En ellas investigadores con gran experiencia evalúan en profundidad los artículos que reciben. Por un lado, estas evaluaciones se centran en aspectos tan genéricos como:

\section{- Calidad del trabajo}

- Pertinencia de la temática para la revista

- Originalidad

- Claridad de expresión

Por otro lado, los revisores también centran su evaluación en aspectos muy específicos del trabajo, como son:

- Que el artículo disponga de un título informativo

- Que las palabras clave y el resumen sean apropiados

- La adecuación y completitud del estado de la cuestión

- La clara y adecuada explicación de la metodología

- La apropiada presentación de los resultados, la discusión y las conclusiones del trabajo

- La pertinencia de la bibliografía

- La adecuación del texto y las figuras a las normas de formato de la revista

- La adecuación de la bibliografía a las normas de formato de la revista

Tomando en consideración todos estos aspectos los revisores emitirán un resultado de la evaluación, que supone que el trabajo pueda ser:

- Aceptado

- No aceptado

- Aceptado con cambios menores

- Aceptado con cambios mayores

En todos los casos, los evaluadores acompañarán su juicio de comentarios sobre el trabajo, que pueden ir dirigidos al editor, a los autores o a ambos. Tomando en consideración estas valoraciones y comentarios, el editor comunicará a los autores del trabajo la decisión favorable o negativa de publicar el trabajo en la revista. Además, acompañará la decisión de los comentarios y valoraciones emitidos por los revisores.

A continuación, se enumeran algunos comentarios reales realizados por revisores. Algunos de ellos referidos a aspectos indicados en este texto. Además, como puede apreciarse, hay comentarios de todo tipo: muy constructivos, constructivos, e incluso "destructivos". 
- "A new paper would have to be edited by a native English speaker who also understands the subject before being submitted"

- "The first two paragraphs are superfluous"

- "The state-of-the-art section is too short to be useful"

- "The description of X-tool is quite incomplete which makes it impossible to put the evaluation results into context"

- "Cited reference on line 45/46 is Olson, H. A. and Wolfram, D. You have switched given names with surnames"

- "My question given this purpose statement of the paper, is what is new here? Are you contributing to our knowledge base by applying known techniques and known evaluation criteria? It doesn't sound like you will present anything new in this research paper"

En cualquier caso, los comentarios negativos de un revisor nunca deberían desmotivar al investigador. Al fin y al cabo, se trata solo de la opinión de una persona. La experiencia demuestra que, con frecuencia, aspectos que son percibidos muy negativamente por algunos revisores y/o revistas no resultan problemáticos para otros revisores y/o revistas.

Por otro lado, para los autores es muy importante tener en cuenta que la aceptación de un artículo condicionado a cambios menores o mayores no asegura su publicación. En estos casos, hasta que los revisores no consideren que el artículo se ha modificado convenientemente para su publicación, los autores no recibirán la confirmación definitiva de aceptación de la publicación del trabajo.

\section{Respuesta a los revisores de una revista académica}

Una vez revisado el artículo, el editor nos comunicará si la revista está interesada o no en su publicación. Si la valoración de los revisores es positiva el editor nos comunicará que el trabajo:

- ha sido aceptado sin cambios -lo cual ocurre rara vez-;

- requiere cambios menores para su publicación -lo que ocurre con frecuencia-; o que

- necesita cambios significativos para su publicación. En este caso tendremos que sopesar si es más conveniente introducir dichos cambios, o declinar la propuesta de la revista y enviar el trabajo a otra publicación.

Si el editor nos comunica que el artículo enviado ha sido aceptado sujeto a modificaciones y se opta por aceptarlas y continuar adelante con su publicación, convendría que los autores siguieran las siguientes pautas o premisas de respuesta a los revisores:

1. Mostrar agradecimiento a los revisores por su comentarios y propuestas para la mejora del artículo. Téngase en cuenta que las observaciones de los revisores casi siempre contribuyen a una mejora sustancial del trabajo. Además, la revisión es una tarea realizada por expertos de manera desinteresada.

2. Se debe responder a todos los comentarios realizados por cada uno de los revisores.

3. Se puede disentir de los revisores, pero justificando rigurosamente nuestra posición.

Si la revista no facilita un formato o un formulario de respuesta, una buena opción sería responder a los revisores utilizando una tabla que recoja, en una columna, los comentarios de los revisores, y en la columna contigua, la respuesta a cada uno de esos comentarios. Además, la respuesta debería explicitar:

- si se acepta o no el cambio propuesto por el revisor $\mathrm{y}$, en caso afirmativo...

- qué cambios se han introducido en el trabajo a partir del comentario o la sugerencia del revisor.

En la figura 4 se muestra una tabla de ejemplo que sigue esta dinámica de respuesta.

Cuando esté cumplimentado, el documento de respuesta será enviado al editor, que a su vez lo trasladará a los revisores del trabajo. A partir de este momento, se pueden dar tres circunstancias:

1. Que el trabajo sea aceptado: porque los revisores consideren que las modificaciones introducidas dotan al trabajo de la calidad necesaria para su publicación.

2. Que el trabajo sea rechazado: porque los revisores consideren que los autores no han conseguido dotar al trabajo de la calidad o el enfoque apropiados para su publicación.

3. Que se inicie una nueva ronda de comentarios o sugerencias que deberán ser respondidos nuevamente por los autores.

En cualquier caso, este proceso culminará con la aceptación o el rechazo del artículo evaluado.

\section{Aceptación y promoción del artículo}

Una vez aceptado el trabajo, la revista procederá a su maquetación y publicación. Es habitual que la revista solicite a los autores una breve biografía y, en algunos casos, una fotografía actualizada que se introducirán en la versión definitiva del artículo. 


\begin{tabular}{|c|c|c|}
\hline $\mathbf{X}$. & Comentaries & Respuesta \\
\hline \multicolumn{3}{|c|}{$\begin{array}{ll}\text { REVISOR } 1 \\
\end{array}$} \\
\hline e1 & 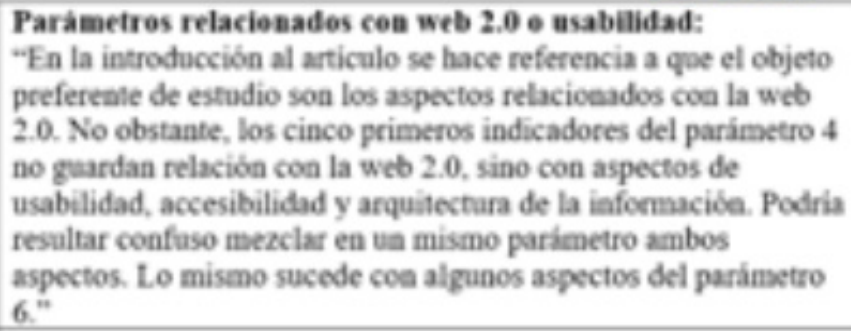 & $\begin{array}{l}\text { Aceptade. } \\
\text { Medificaciéa: Se han realizado aclaraciones en la } \\
\text { introducción y otros cambios en el titulo para } \\
\text { evitar los posibles malentendidos sellalados por el } \\
\text { evaluador. En la introducción se razooa el motivo } \\
\text { de mantener esta clase de indicadores y se ha } \\
\text { modificado el título para que responda a esta } \\
\text { visión más amplia. }\end{array}$ \\
\hline$\theta 2$ & $\ldots$ & $\ldots$ \\
\hline 63 & $\ldots$ & $\ldots$ \\
\hline \multicolumn{3}{|c|}{ REVISOR DOS } \\
\hline e1 & $\begin{array}{l}\text { Coefusiba entre los parámetros } 8 \text { y 9: } \\
\text { "Los parimetros } 8 \text { y } 9 \text { recogen aspectos may similares, que en } \\
\text { algunos casos puede llevar tambièn a confusióa. El parimetro } 9 \\
\text { (empleo de redes sociales) podria agrupar también los } \\
\text { indicadores del parimetro } 8 \text {." }\end{array}$ & $\begin{array}{l}\text { Aceptade. } \\
\text { Modificacién: De acuerdo con lo que señala el } \\
\text { reviser, algunos de los indicadores que aparecen } \\
\text { en el parimetro } 8 \text { pueden formar parte del } \\
\text { parimetro } 9 . \\
\text { Por esta razón, se han unificado todos los } \\
\text { indicadores a los que se hace referencia en un } \\
\text { único parametro, el parimetro } 8\end{array}$ \\
\hline 02 & $\ldots$ & $\ldots$. \\
\hline
\end{tabular}

Figura 4. Ejemplo de tabla de respuesta a los comentarios de los revisores de un artículo. Fuente: elaboración propia.

Publicado el trabajo, a los autores les espera la ardua tarea de promocionar su trabajo. Pueden hacerlo de diversas maneras. Si se ha publicado en la modalidad Open Access se podrá distribuir el trabajo a través de diferentes repositorios y plataformas. Si no ha sido así, los autores tendrán que consultar en la revista si pueden difundir el pre-print o post-print de su artículo.

$\mathrm{Si}$ disponemos de un documento que podamos distribuir libremente, los siguientes serán espacios perfectos para dar a conocer y promocionar nuestro trabajo: los repositorios de instituciones académicas -especialmente de la nuestra-, las redes sociales académicas, los servicios de alojamiento de documentos con contenido de carácter académico o técnico, o nuestra web personal.

Si no tenemos la posibilidad de distribuir el artículo, siempre podremos darlo a conocer referenciándolo -y enlazándolo con la página web de la revista en la que aparece- en las plataformas anteriormente mencionadas.

Otras estrategias adicionales son, por ejemplo, el envío del documento a todos los autores que se han citado en nuestro artículo y, por supuesto, anunciar su publicación en las plataformas sociales que utilizamos habitualmente.

\section{Conclusiones}

Como acabamos de comprobar, son muchos los aspectos que un autor ha de tomar en consideración para maximizar sus posibilidades de publicar con éxito en una revista científica. En este documento se han intentado recopilar algunas sugerencias, recomendaciones y buenas prácticas que ayuden a aquellas personas que se inician en el mundo de la publicación académica y, más concretamente, en la publicación de artículos de investigación.

Especialmente relevantes son las figuras y tablas presentadas en el texto. Con ellas se ha pretendido facilitar a los investigadores noveles herramientas, no siempre fáciles de encontrar o elaborar, que les ayuden a seleccionar revistas, enviar sus artículos y responder a los revisores.

Terminamos con una breve reflexión sobre el contexto académico, en el que actualmente la publicación de artículos en revistas científicas se ha convertido en un requerimiento, no solo para desarrollar una carrera académica, sino incluso para la superación de diferentes programas de postgrado. Por ello, consideramos muy necesarios textos que, como este, faciliten pautas que guíen a los nuevos investigadores en la difícil tarea de publicar.

\section{Referencias}

Buenz, E. J. (2019). Essential elements for high-impact scientific writing. Nature. [Accesible en: https://go.nature. com/2ttaayp]

Codina, Lluís (2018). Artículos científicos: quién puede firmarlos y en qué orden. Ética y pragmatismo de la publicación académica. [Accesible en: https://www. 
lluiscodina.com/etica-publicacion-academica/]

Committee on Publication Ethics (COPE). Resources about authorship and contributorship. [Accesible en: https:// publicationethics.org/authorship]

Day, R. A. (1998). How to write and publish scientific papers.

Gustavii, B. (2017). How to write and illustrate a scientific paper. Cambridge University Press.

Horta, H., \& Santos, J. M. (2016). The impact of publishing during $\mathrm{PhD}$ studies on career research publication, visibility, and collaborations. Research in Higher Education, 57(1), 28-50.

Mauvais-Jarvis, F. (2016). Developing Academic Visibility in the Medical Sciences. The Ochsner Journal, 16(3), 208.

Pérez-Altable, L. (2016). Social movements and network analysis: the case of Tunisia digital activism before and during the Arab Spring (2010-2011) [Doctoral dissertation], Universitat Pompeu Fabra.

Seibert, S. E., Kacmar, K. M., Kraimer, M. L., Downes, P. E., \& Noble, D. (2017). The role of research strategies and professional networks in management scholars' productivity. Journal of Management, 43(4), 1103-1130.

Sestak, Z. (2003). How to write a paper. G. M. Hall (Ed.). BMJ.

Wee, B. V., \& Banister, D. (2016). How to write a literature review paper?. Transport Reviews, 36(2), 278-288. 

ISBN 978-84-09-20524-0

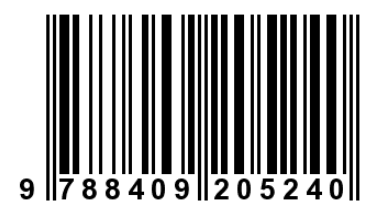

Máster Universitario en Investigación en Comunicación Social (MUCS)

\begin{tabular}{l|ll}
$\mathbf{u} p f$. & $\begin{array}{l}\text { Universitat } \\
\text { Pompeu Fabra } \\
\text { Barcelona }\end{array}$ & $\begin{array}{l}\text { DIGIDOC Grup de Recerca } \\
\text { en Documentació Digital } \\
\text { i Comunicació Interactiva }\end{array}$
\end{tabular} 